\title{
Pregnancy massage reduces prematurity, low birthweight and postpartum depression
}

\author{
Tiffany Field ${ }^{\mathrm{a}, \mathrm{b}, *}{ }^{a}$, Miguel Diego $^{\mathrm{a}}$, Maria Hernandez-Reif ${ }^{\mathrm{c}}$, \\ Osvelia Deeds ${ }^{a}$, Barbara Figueiredo ${ }^{d}$ \\ a Touch Research Institutes, University of Miami Medical School, United States \\ b Fielding Graduate University, United States \\ c University of Alabama, United States \\ d University of Portugal, Portugal
}

\section{A R T I C L E I N F O}

\section{Article history:}

Received 18 June 2008

Received in revised form 6 February 2009

Accepted 7 July 2009

\section{Keywords:}

Pregnancy massage

Prematurity

Low birthweight

Postpartum depression

\begin{abstract}
A B S T R A C T
Pregnant women diagnosed with major depression were given 12 weeks of twice per week massage therapy by their significant other or only standard treatment as a control group. The massage therapy group women versus the control group women not only had reduced depression by the end of the therapy period, but they also had reduced depression and cortisol levels during the postpartum period. Their newborns were also less likely to be born prematurely and low birthweight, and they had lower cortisol levels and performed better on the Brazelton Neonatal Behavioral Assessment habituation, orientation and motor scales.
\end{abstract}

(C) 2009 Elsevier Inc. All rights reserved.

\section{Introduction}

\subsection{Prenatal depression}

Prenatal depression has been noted in 10-25\% of pregnant women (Gordon, Cardone, Kim, Gordon, \& Silver, 2006; Kim et al., 2006) and it is a potential risk factor for many adverse perinatal complications including a greater incidence of prematurity (Field, Diego, Dieter et al., 2004; Orr, James, \& Blackmore Prince, 2002) and low birthweight (<2500 g) (Field, Diego, Dieter et al., 2004; Oberlander, Warburton, Misri, Aghajanian, \& Hertzman, 2006), as well as more frequent intensive care at birth (Chung, Lau, Yip, Chiu, \& Lee, 2001; Oberlander et al., 2006). Prenatal depression also contributes to biobehavioral dysregulation in the fetus marked by greater activity levels (Dieter et al., 2001), elevated resting heart rate (Allister, Lester, Carr, \& Liu, 2001), and increased heart rate reactivity (Monk et al., 2004).

Neonates of prenatally depressed women are also more likely to exhibit biobehavioral dysregulation (Field, Diego, Dieter et al., 2004; Lundy et al., 1999), and during infancy they are more likely to show mental, motor and emotional delays (Patel, DeSouza, \& Rodrigues, 2003) and growth delays (Patel et al., 2003; Rahman, Lovel, Bunn, Iqbal, \& Harrington, 2004). Later, as children, they more often have emotional problems (Luoma et al., 2001).

* Corresponding author at: Touch Research Institute, University of Miami School of Medicine, PO Box 016820, Miami, FL 33101, United States. Tel.: +1 3052436781 .

E-mail address: tfield@med.miami.edu (T. Field). 


\subsection{Prenatal interventions}

Depression in non-pregnant women is effectively treated using antidepressant medications (APA, 2000), but antidepressants can pass through the placenta (Hendrick, Stowe et al., 2003) and have adverse effects including premature delivery, low birthweight (Field, 2008; Hendrick, Smith et al., 2003) and neonatal dysregulation (Oberlander et al., 2006; Zeskind \& Stephens, 2004). Psychotherapy is also an effective treatment for depression (APA, 2000) and one that does not pose the risks of antidepressants. However, psychotherapy has not been assessed for its effects on pregnancy complications and neonatal outcomes. In addition, low socioeconomic status women are less likely to have access to and receive treatment for depression (US Department of Health and Human Services, 2001).

Other non-pharmacological interventions for prenatal depression have also been investigated such as massage therapy. Massage therapy is a popular alternative treatment that involves manipulation of the soft tissues of the body through pressure and movement. Massage therapy may provide an optimal alternative intervention for treating prenatal depression and improving perinatal outcomes, as it may be effectively administered by a significant other. Women who are massaged during pregnancy report fewer depression and anxiety symptoms, better sleep, less back pain, and they have lower cortisol and norepinephrine levels by the last day of the study irrespective of whether the massage is administered by a professional massage therapist or their partner (Field, Diego, \& Hernandez-Reif, 2007).

\subsection{Massage therapy reduces prenatal depression}

Massage therapy reduces prenatal depression and decreases salivary and urinary cortisol levels. In our first study on massage with pregnant women, massage therapy and relaxation therapy groups were compared (Field et al., 1999). After 20-min sessions twice per week for 5 weeks, the massage therapy group reported less depressed mood, and they had fewer perinatal complications.

In a subsequent study, we recruited clinically depressed pregnant women and randomly assigned them to a massage therapy, relaxation therapy or control group and compared them to each other and to a non-depressed group at the end of the pregnancy (Field, Diego, Hernandez-Reif, Schanberg, \& Kuhn, 2004). The massage and relaxation therapy groups received two 20-min therapy sessions per week by their significant other for the last trimester of pregnancy. Once again the massaged women had less depressed mood and disturbed sleep patterns, as well as less pain, lower stress hormones and fewer perinatal complications including a lower incidence of prematurity.

The purpose of the present study was to assess the effects of pregnancy massage by significant others on prenatal measures as well as perinatal outcomes and in a larger sample. Thus, the aims of this study were: (1) to replicate our previous studies on the effects of pregnancy massage on prenatal measures (Field et al., 1999; Field, Diego, Hernandez-Reif et al., 2004) and (2) to add measures of postnatal outcomes including postpartum depression and neonatal measures including cortisol and the Brazelton Behavioral Neonatal Assessment Scale (Brazelton, 1973). Massage therapy was expected to reduce prenatal depression, anxiety, anger, daily hassles, back pain and sleep disturbances in the women and the incidence of prematurity and low birthweight as it has done in previous studies (Field et al., 1999; Field, Diego, Hernandez-Reif et al., 2004) and to reduce postpartum depression and neonatal dysregulation.

\section{Method}

\subsection{Participants}

Two hundred depressed women were recruited between 16 and 20 weeks gestation from two ultrasound clinics $(M=19.2$ weeks). The women averaged 26.2 years of age, were primarily low socioeconomic status ( $M=3.9$ on the Hollingshead SES Index), and were distributed approximately 57\% Hispanic, 38\% African-American, and 5\% Non-Hispanic. The groups did not differ on these demographic variables (see Table 1). Twenty percent of the women who were interviewed met SCID criteria for major depressive disorder that had persisted across pregnancy to the time of recruitment.

Pregnant women were considered eligible for recruitment if they were low risk pregnancies and met the following criteria: (a) gestational age (GA) between 16 and 20 weeks; (b) 18-30 years of age; (c) singleton pregnancy; (d) uncomplicated pregnancy; (e) major depression across pregnancy; and (f) living with a partner. Mothers were specifically excluded if they: (a) had HIV or any other infectious disease; (b) had hypertension; (c) exhibited any pregnancy complications prior to recruitment including: gestational diabetes, ectopic pregnancies, anemia, placenta previa, placental abruption or preeclampsia; (d) suffered from any metabolic or eating disorder (obesity, bulimia, anorexia); (e) were currently using medications other than vitamins; or (f) had smoked cigarettes or used drugs during their pregnancy.

Gestational age was estimated based on the mothers' last menstrual period (LMP). Only mothers who were able to accurately recall their LMP date were recruited for the study. Any women with suicidal ideation, bipolar disorder, schizophrenia or any psychiatric disorder other than MDD were excluded from the study. All women with MDD and these other disorders were referred to our collaborating psychiatrist. 


\subsection{Procedures}

Following informed consent, the women were assessed for depression using the SCID and were recruited only if they had experienced depression throughout their 16-20 weeks of pregnancy. The women were then randomly assigned to a massage or a standard treatment control group. The women in the massage group received 2 moderate pressure massages per week for a period of 12 weeks by their significant others who were taught the massage by a massage therapist and were given DVDs for at-home coaching in the massage procedure.

\subsection{Pregnancy massage}

The massage therapy began at 20 weeks gestation and continued until 32 weeks gestation (when the second assessment was made). Each session began with the mother in a side-lying position, with pillows positioned behind her back and between her legs for support. The massage is administered in the following sequence and then repeated with the mother lying on her other side (10 min each side for a total of $20 \mathrm{~min}$ ): (1) Head and neck: massaging the scalp, making small circles from the forehead along the hairline and down to the temples, and kneading the neck from the base up; (2) Back: using the heels of the hands, moving along the spine; using the palms moving the hands with rocking movements from the top of the shoulder blades to the backbone; pressing fingertips along both sides of the spine from the neck to the backbone and then stroking upward from the hips to the neck; stroking the shoulder muscles (trapezius); inching up the back, using fingertips placed on the sides of the spine, starting from the hipbone to the neck and then reversing the direction downward using fingertips in a raking fashion; massaging the lower back from the backbone across the waistline using the heels of the hands to make large circles; long gliding strokes from the hip up and over the shoulders; (3) Arms: making long sweeping strokes from the elbow up and over the shoulder; kneading the muscles from above the elbow to the shoulder; stroking from the wrist to the elbow; kneading the muscles between the wrist and the elbow; (4) Hands: massaging the hand using thumbs to make small circles on the palm; on the back of the hand, rubbing between the spaces of the bones; sliding down each finger; (5) Legs: long sweeping strokes from the knee to the thigh, up and over the hip; kneading the muscles between the knee and thigh; long sweeping strokes from the ankle up towards the knee; kneading the muscles between the ankle and knee; sliding the hand from the Achilles tendon up towards the upper calf and sliding down to the heel with less pressure several times; (6) Feet: massaging the soles from the toes to the heel with fingers and thumbs and moving back towards the toes; sliding down each toe and rotating the toes three times; stroking the top of the foot towards the leg. This same pregnancy massage procedure was effective in reducing depression and improving perinatal outcomes in depressed pregnant women (Field, Diego, Hernandez-Reif et al., 2004).

\subsection{Assessments}

(1) Maternal Questionnaires. After completing the informed consent at 16-20 weeks gestational age, the women were administered: (a) the Sociodemographic Questionnaire; (b) the Structured Clinical Interview for DSM-IV Axis I Disorders (SCID); (c) the Center for Epidemiological Studies-Depression (CES-D) Scale; (d) the State Anxiety Inventory (STAI); (e) the State Anger Inventory (STAXI); (f) the Daily Hassles Scale; (g) the Sleep Disturbances Scale; and (h) the Back Pain Scale. The Spanish versions of these scales were given to the Spanish-only speaking women. The women were given the SCID and the CES-D again during the second assessment ( 32 weeks GA) and during the first 2 days following delivery.

(a) Sociodemographic Questionnaire. The Sociodemographic Questionnaire is comprised of 11 items on age, parity, education, occupation, income and length of time couples were in the relationship, marital status, ethnicity and social support. The Questionnaire also contains all the inclusion/exclusion criteria.

(b) Structured Clinical Interview for DSM-IV Axis I Disorders (SCID-D). All women in the study were administered the SCID-I (Non-patient edition: research version) to determine major depression and to screen out other disorders including substance use, anxiety disorder, bipolar disorder, schizophrenia and other psychotic disorders. Although depression is often comorbid with anxiety and substance use, these groups were excluded because of their potentially confounding depression effects in this study. Spanish-only speaking women were given the Spanish version of the SCID. Only women who met criteria for major depressive disorder (MDD) throughout pregnancy to date and women who did not meet criteria for any other disorder were eligible for the study. The SCID was administered by the psychology research associates following training and with continuing supervision by a clinical psychologist.

(c) The Center for Epidemiological Studies-Depression Scale (CES-D; Radloff, 1977). This 20-item scale was included to assess the symptoms of depression. The woman is asked to report on her feelings during the preceding week. The scale has adequate test/retest reliability (.60 over several weeks), internal consistency (.80-.90) and concurrent validity (Wells, Klerman, \& Deykin, 1987). Test-retest reliability over a 1-month period on this sample was .79, suggesting some short-term stability of depressive symptoms (Radloff, 1991).

(d) The State Anxiety Inventory (STAI) is comprised of 20 items and assesses the intensity of anxiety symptoms. The scores range from 20 to 90 , and the cutoff for high anxiety is 48 . Research has demonstrated that the STAI has adequate concurrent validity and internal consistency (Spielberger, Gorsuch, \& Lushene, 1970), and the scale has been used in several studies with pregnant women (e.g., Da Costa, Larouche, Drista, \& Brender, 2000). 
Table 1

Means for demographic variables for depressed control versus depressed massage groups (standard deviations in parentheses).

\begin{tabular}{lcc}
\hline Variables & Groups & \\
\cline { 2 - 3 } & Control & Massage \\
\hline Age & $25.2(5.8)$ & $27.2(6.5)$ \\
SES & $3.9(1.1)$ & $3.8(.9)$ \\
Parity & $1.2(1.2)$ & $1.1(1.2)$ \\
Ethnicity (\%) & & \\
$\quad$ Hispanic & 57 & 58 \\
Black & 40 & 35 \\
Caucasian & 3 & 7 \\
Stressful situation (\%) & 59 & 60 \\
Medical complications (\%) & 22 & 18 \\
Medications (\%) & 19 & 24 \\
\hline
\end{tabular}

(e) The State Anger Inventory (STAXI) (Spielberger, Ritterband, Sydeman, Reheiser, \& Unger, 1995) is a 10-item inventory that assesses general feelings of anger based on a 4-point Likert scale ranging from 1 (almost never) to 4 (almost always). Typical questions include "I am quick tempered" and "I fly off the handle". Psychometric properties have been established for the STAXI on diverse ethnic groups including a reliability coefficient of .97 .

(f) The Daily Hassles Scale (Field et al., 2003). This scale was developed to assess the hassles being experienced by expectant mothers. The 16 items on a 4-point Likert scale include questions on people such as family members, landlords, friends and finances creating hassles.

(g) The Sleep Disturbance Scale (Verran \& Snyder-Halpern, 1988). Questions on this 15-item scale are rated on a visual analog anchored at one end with effective sleep responses (e.g., "Did not awaken," "Had no trouble sleeping") and at the opposite end with ineffective responses (e.g., "Was awake 10 hours," "Had a lot of trouble falling asleep"). The participants place a mark across the answer line at the point that best reflects their last night's sleep. A reliability coefficient of .82 has been reported for this scale (Verran \& Snyder-Halpern, 1988).

(h) Back Pain. Participants completed pre- and post-session VITAS pain scales with reference to back pain on the first and last days of the study. Pain perception is rated on a visual analog scale ranging from 0 (no pain) to 10 (worst possible pain), and anchored with 5 faces. The faces, located at two point intervals, range from very happy (0), to happy (2), contented (4), somewhat distressed (6), distressed (8), and very distressed (10).

(i) Newborn Measures. These were taken during the first 2 days following the delivery and included gestational age, birthweight, saliva cortisol levels and the Brazelton Neonatal Behavior Assessment Scale.

(j) Saliva Cortisol. Filter paper was placed on the tongue for $60 \mathrm{~s}$ for saliva collection. Samples were taken at approximately 10 a.m. just before Brazelton Scale Assessment. The samples were frozen and later sent to Salimetrics for assays. To assay cortisol, the saliva is extracted from the filter strip in $0.5 \mathrm{ml}$ of assay buffer by shaking for $24 \mathrm{~h}$. This extraction procedure dilutes the saliva 1:5. Cortisol concentration in the buffer is determined using a high sensitivity ElA kit (Salimetrics) that detects cortisol levels in the range of $0.007-1.8 \mu \mathrm{g} / \mathrm{dl}$. Briefly, $50 \mu \mathrm{g}$ of extraction buffer is added in duplicate to the assay. Standard curves are fitted by weighted regression analysis and unknown values are computed. The detection limit of samples collected in this manner is $0.035 \mu \mathrm{g} / \mathrm{dl}$.

(k) Brazelton Neonatal Behavior Assessment Scale (BNBAS). The Brazelton Neonatal Behavior Assessment Scale (Brazelton, 1973 ) is administered midway between feedings. The scale is comprised of 20 neurological reflex items and 27 other items summarized according to 7 factors: habituation, orientation, motor behavior, range of state, state regulation, autonomic stability and abnormal reflexes. In addition, excitability and depressive behavior are recorded. The Brazelton examiners are unaware of the infant's group assignments and are trained to a .90 reliability criterion prior to the study. This assessment was included because lower orientation and motor scores and more depressive behaviors were noted on the Brazelton scale in an earlier study on newborns of depressed mothers (Lundy et al., 1999).

\section{Results}

\subsection{Demographic variables}

Following a $30 \%$ attrition rate, the sample sizes were 88 in the massage group and 61 in the control group. ANOVAs and Chi Squares on demographic variables revealed no group differences on demographic variables including maternal age, SES, parity, ethnicity, experiencing a stressful situation during pregnancy, medical complications and medications (see Table 1). 
Table 2

Means for women's self-report measures from first and last prenatal assessments for depressed control versus depressed massage groups (standard deviations in parentheses).

\begin{tabular}{|c|c|c|c|c|c|c|c|}
\hline \multirow[t]{3}{*}{ Variable } & \multicolumn{4}{|l|}{ Groups } & \multirow[t]{3}{*}{ Effect } & \multirow[t]{3}{*}{$F$} & \multirow[t]{3}{*}{$p$} \\
\hline & \multicolumn{2}{|l|}{ Control } & \multicolumn{2}{|l|}{ Massage } & & & \\
\hline & First & Last & First & Last & & & \\
\hline Depression (CES-D) & $23.7(7.7)$ & $21.5(10.0)$ & $20.3(9.1)$ & $14.8(8.5)$ & Group $\times$ time & 7.72 & .007 \\
\hline Anxiety (STAI) & $41.8(10.0)$ & $41.0(9.4)$ & $41.3(9.1)$ & $39.2(10.6)$ & & & NS \\
\hline Anger (STAXI) & $22.4(8.7)$ & $20.2(6.1)$ & $19.4(6.6)$ & $17.7(6.3)$ & & & NS \\
\hline Daily hassles & $24.5(7.3)$ & $23.9(7.3)$ & $22.9(6.0)$ & $22.5(7.2)$ & & & NS \\
\hline Back pain & $4.2(2.4)$ & $5.0(2.6)$ & $4.5(3.0)$ & $4.1(3.0)$ & Group $\times$ time & 4.87 & .03 \\
\hline Sleep disturbances & $49.9(19.4)$ & $55.7(18.5)$ & $48.4(22.4)$ & $47.3(22.4)$ & & & NS \\
\hline
\end{tabular}

Table 3

Means for variables for depressed control versus depressed massage groups (standard deviations in parentheses).

\begin{tabular}{|c|c|c|c|c|}
\hline \multirow[t]{2}{*}{ Variables } & \multicolumn{2}{|l|}{ Groups } & \multirow[t]{2}{*}{$F$} & \multirow[t]{2}{*}{$p$} \\
\hline & Control & Massage & & \\
\hline Gestational age & $38.7(1.6)$ & $39.0(1.2)$ & 2.86 & .09 \\
\hline Birthweight & $3225.5(491.6)$ & 3317.5 (360.4) & 1.99 & NS \\
\hline \multirow[t]{2}{*}{ Variables } & \multicolumn{2}{|l|}{ Groups } & \multirow[t]{2}{*}{$X^{2}$} & \multirow[t]{2}{*}{$p$} \\
\hline & Control & Massage & & \\
\hline Incidence prematurity (\%) & 16.4 & 4.0 & 8.40 & .004 \\
\hline Incidence low birthweight (\%) & 10.0 & 2.0 & 5.70 & .02 \\
\hline
\end{tabular}

Table 4

Means for newborn cortisol levels and Brazelton Neonatal Behavior Assessment Scale Scores for depressed control versus depressed massage groups (standard deviations in parentheses).

\begin{tabular}{|c|c|c|c|c|}
\hline \multirow[t]{2}{*}{ Variables } & \multicolumn{2}{|l|}{ Groups } & \multirow[t]{2}{*}{$F$} & \multirow[t]{2}{*}{$p$} \\
\hline & Control & Massage & & \\
\hline Cortisol levels & $502.5(251.2)$ & $261.3(214.9)$ & 6.24 & .02 \\
\hline \multicolumn{5}{|l|}{ Brazelton Scales } \\
\hline Habituation & $4.6(2.3)$ & $5.7(1.4)$ & 5.12 & .03 \\
\hline Orientation & $5.0(1.5)$ & $5.6(1.2)$ & 4.61 & .05 \\
\hline Motor & $4.6(1.2)$ & $5.3(.6)$ & 12.17 & .001 \\
\hline State organization & $3.7(1.1)$ & $3.8(.8)$ & & NS \\
\hline State regulation & $5.9(2.8)$ & $5.4(1.2)$ & & NS \\
\hline Autonomic stability & $5.5(1.4)$ & $6.0(1.2)$ & & NS \\
\hline Reflexes & $3.2(3.3)$ & $2.3(2.0)$ & & NS \\
\hline Depression & $2.8(2.0)$ & $2.1(1.4)$ & & .05 \\
\hline
\end{tabular}

\subsection{Self-report measures}

Repeated measures ANOVAs on self-report measures revealed the following (see Table 2): (1) on depression (CES-D score), a group by time interaction effect (first to last prenatal assessment) suggested that the massage group had a greater decrease in depression scores than the control group; (2) on anxiety, anger, hassles and sleep disturbance scores, no group or group by trials interaction effects were noted; and (3) on back pain, the massage group showed a decrease in back pain, while the control group showed an increase in back pain across the prenatal period.

\subsection{Neonatal outcomes}

As can be seen in Table 3, ANOVAs on neonatal outcome measures revealed the following: (1) the massage group infants tended to be greater gestational age; (2) the groups did not differ on birthweight; (3) the massage group experienced a lower rate of prematurity; and (4) the massage group had a lower incidence of low birthweight.

\subsection{Neonatal Behavior Assessment Scale scores}

As can be seen in Table 4, the massage group neonates received higher scores on the Brazelton Neonatal Behavior Assessment Scales including: (1) habituation; (2) orientation; (3) motor; and (4) depression scores. In addition, they had lower cortisol levels. 


\subsection{Postpartum depression and cortisol levels}

The massage group mothers had lower postpartum depression scores than the control mothers $(M=14.2$ versus 24.9 , $F=6.83, p=.01)$. They also had lower cortisol levels $(M=128.6$ versus $2328.8, F=4.17, p=.05)$.

\section{Discussion}

The higher than national average prematurity and low birthweight rates for the depressed mothers in this sample were not surprising given previous reports on a greater incidence of prematurity in depressed women (Field, Diego, Dieter et al., 2004; Oberlander et al., 2006; Orr et al., 2002). The decreased depression and low back pain in the massaged women and their fewer prenatal complications including a 75\% lower prematurity rate and an 80\% lower incidence of low birthweight in the massaged depressed women were also not surprising given our previous finding of a lower prematurity rate in depressed women given pregnancy massage (Field, Diego, Hernandez-Reif et al., 2004).

These findings were unique in that postpartum depression and cortisol levels were decreased in the pregnancy massage women. The decrease in depression by week 32 of gestation in the massaged women persisted through the duration of pregnancy. A follow-up assessment would be necessary to determine whether these women remained free of postpartum depression into infancy. Nonetheless, to have mothers less depressed during the neonatal period would likely enhance their bonding to their infants. Having more mature and more interactive newborns would also certainly enhance that process (Field, Diego, \& Hernandez-Reif, 2006).

That the newborns of the massaged mothers also had lower cortisol levels than the newborns of the control mothers may relate to the mothers' lower cortisol levels and their decreasing depression across pregnancy. Although this is the first report of newborns' cortisol levels being lower after their mothers received prenatal massage, the reduction in cortisol following massage has been reported in many studies (see Field, Hernandez-Reif, Diego, Schanberg, \& Kuhn, 2005 for a review).

The more optimal Brazelton scores in the newborns of prenatally massaged mothers are perhaps not surprising in light of their lower cortisol levels. In addition, the better performances on habituation, orientation, motor and depression subscale scores are consistent with data from our previous study (Field, Diego, Hernandez-Reif et al., 2004).

These data highlight the therapeutic efficacy of an early cost-effective intervention for prenatally depressed women and their offspring. The intervention not only improved the pregnancy and postpartum course for the women and infants but would be expected to help their significant others. Massage by significant others has helped by lowering their anxiety levels (Latifses, Bendell Estroff, Field, \& Bush, 2005) and their stress hormones (Field, Hernandez-Reif, Quintino, Schanberg, \& Kuhn, 1998).

In summary, pregnant women diagnosed with major depression were given 12 weeks of twice per week massage therapy by their significant other or only standard treatment as a control group. The massage therapy group women versus the control group women not only had reduced depression by the end of the therapy period, but they also had reduced depression and cortisol levels during the postpartum period. Their newborns were also less likely to be born prematurely and low birthweight, and they had lower cortisol levels and performed better on the Brazelton Neonatal Behavioral Assessment habituation, orientation, motor and depression scales.

\section{Acknowledgments}

We would like to thank the women, men and infants who participated in this study and the research associates who assisted us. This research was supported by a Merit Award (MH46586), Senior Research Scientist Awards (MH00331 and AT001585) and a March of Dimes Grant (\# 12-FYO3-48) to Tiffany Field and funding from Johnson and Johnson Pediatric Institute to the Touch Research Institute.

\section{References}

Allister, L., Lester, B. M., Carr, S., \& Liu, J. (2001). The effects of maternal depression on fetal heart rate response to vibroacoustic stimulation. Developmental Neuropsychology, 20, 639-651.

American Psychiatric Association. (2000). Practice guideline for the treatment of patients with major depressive disorder (revision). American Journal of Psychiatry, 157, 1-45.

Brazelton, T. B. (1973). Neonatal behavioral assessment scale.

Chung, T. K., Lau, T. K., Yip, A. S., Chiu, H. F., \& Lee, D. T. (2001). Antepartum depressive symptomatology is associated with adverse obstetric and neonatal outcomes. Psychosomatic Medicine, 63, 830-834.

Da Costa, D., Larouche, J., Drista, M., \& Brender, W. (2000). Variation in stress levels over the course of pregnancy: Factors associated with elevated hassles, state anxiety and pregnancy-specific stress. Journal of Psychosomatic Research, 47, 609-621.

Dieter, J. N. I., Field, T., Hernandez-Reif, M., Jones, N. A., LeCanuet, J. P., Salman, F. A., et al. (2001). Maternal depression and increased fetal activity. Journal of Obstetrics and Gynaecology, 21, 468-473.

Field, T. (2008). Prematurity and potential predictors. International Journal of Neuroscience, 118, 277-289.

Field, T., Diego, M., Dieter, J., Hernandez-Reif, M., Schanberg, S., Kuhn, C., et al. (2004). Prenatal depression effects on the fetus and the newborn. Infant Behavior and Development, 27, 216-229.

Field, T., Diego, M., \& Hernandez-Reif, M. (2006). Prenatal depression effects on the fetus and newborn: A review. Infant Behavior and Development, 29, $445-455$

Field, T., Diego, M., \& Hernandez-Reif, M. (2007). Massage therapy research. Developmental Review, 27, 75-89. 
Field, T., Diego, M., Hernandez-Reif, M., Schanberg, S., \& Kuhn, C. (2004). Massage therapy effects on depressed pregnant women. Journal of Psychosomatic Obstetrics \& Gynecology, 25, 115-122.

Field, T., Diego, M., Hernandez-Reif, M., Schanberg, S., Kuhn, C., Yando, R., et al. (2003). Pregnancy anxiety and comorbid depression and anger effects on the fetus and neonate. Depression and Anxiety, 17, 140-151.

Field, T., Hernandez-Reif, M., Diego, M., Schanberg, S., \& Kuhn, C. (2005). Cortisol decreases and serotonin and dopamine increase following massage therapy. International Journal of Neuroscience, 115, 1397-1413.

Field, T., Hernandez-Reif, M., Hart, S., Theakston, H., Schanberg, S., Kuhn, C., et al. (1999). Pregnant women benefit from massage therapy. Journal of Psychosomatic Obstetrics E' Gynecology, 20, 31-38.

Field, T., Hernandez-Reif, M., Quintino, O., Schanberg, S., \& Kuhn, C. (1998). Elder retired volunteers benefit from giving massage therapy to infants. Journal of Applied Gerontology, 17, 229-239.

Gordon, T. E., Cardone, I. A., Kim, J. J., Gordon, S. M., \& Silver, R. K. (2006). Universal perinatal depression screening in an Academic Medical Center. Obstetrics and Gynecology, 107, 342-347.

Hendrick, V., Smith, L. M., Suri, R., Hwang, S., Haynes, D., \& Altshuler, L. (2003). Birth outcomes after prenatal exposure to antidepressant medication. American Journal of Obstetrics and Gynecology, 188, 812-815.

Hendrick, V., Stowe, Z. N., Altshuler, L. L., Hwang, S., Lee, E., \& Haynes, D. (2003). Placental passage of antidepressant medications. The American Journal of Psychiatry, 160, 993-996.

Kim, H. G., Mandell, M., Crandall, C., Kuskowski, M. A., Dieperink, B., \& Buchberger, R. L. (2006). Antenatal psychiatric illness and adequacy of prenatal care in an ethnically diverse inner-city obstetric population. Archives of Women's Mental Health, 9, 103-107.

Latifses, V., Bendell Estroff, D., Field, T., \& Bush, J. (2005). Fathers massaging and relaxing their pregnant wives lowered anxiety and facilitated marital adjustment. Journal of Bodywork and Movement Therapies, 9, 277-282.

Lundy, B. L., Jones, N. A., Field, T., Nearing, G., Davalos, M., Pietro, P., et al. (1999). Prepartum depression effects on neonates. Infant Behavior and Development, $22,121-137$.

Luoma, I., Tamminen, T., Kaukonen, P., Laippala, P., Puura, K., Salmelin, R., et al. (2001). Longitudinal study of maternal depressive symptoms and child well-being. Journal of the American Academy of Child and Adolescent Psychiatry, 40, 1367-1374.

Monk, C., Sloan, R. P., Myers, M. M., Ellman, L., Werner, E., Jeon, J., et al. (2004). Fetal heart rate reactivity differs by women's psychiatric status: An early marker for developmental risk? Journal of the American Academy of Child and Adolescent Psychiatry, 43, 283-290.

Oberlander, T. F., Warburton, W., Misri, S., Aghajanian, J., \& Hertzman, C. (2006). Neonatal outcomes after prenatal exposure to selective serotonin reuptake inhibitor antidepressants and maternal depression using population-based linked health data. Archives of General Psychiatry, $63,898-906$.

Orr, S. T., James, S. A., \& Blackmore Prince, C. (2002). Maternal prenatal depressive symptoms and spontaneous preterm births among African-American women in Baltimore, Maryland. American Journal of Epidemiology, 156, 797-802.

Patel, V., DeSouza, N., \& Rodrigues, M. (2003). Postnatal depression and infant growth and development in low income countries: A cohort study from Goa, India. Archives of Diseases in Childhood, 88, 34-37.

Radloff, L. (1991). The use of the Center for Epidemiological Studies Depression Scale in adolescents and young adults. Journal of Youth and Adolescence, 20, $149-165$.

Radloff, L. (1977). The CES-D scale: A self-report depression scale for research in the general population. Applied Psychological Methods, 1, 385-401.

Rahman, A., Lovel, H., Bunn, J., Iqbal, Z., \& Harrington, R. (2004). Mothers' mental health and infant growth: A case-control study from Rawalpindi, Pakistan. Child Care, Health and Development, 30, 21-27.

Spielberger, C., Gorsuch, R., \& Lushene, R. (1970). The state/trait anxiety inventory. Paolo Alto, CA: Consulting Psychology Press

Spielberger, C. D., Ritterband, L. M., Sydeman, S. J., Reheiser, E. C., \& Unger, K. K. (1995). Assessment of emotional states and personality traits: Measuring psychological vital signs. Clinical personality assessment: Practical approaches. New York: Oxford University Press.

US Department of Health and Human Services. (2001). Mental health: Culture, race and ethnicity - A supplement to mental health: A report of the surgeon general. Rockville, MD: US Department of Health and Human Services, Substance Abuse and Mental Health Services Administration, Center for Mental Health Services.

Verran, J. A., \& Snyder-Halpern, R. (1988). Do patients sleep in the hospital? Applied Nursing Research, 1, 95.

Wells, V. E., Klerman, G. L., \& Deykin, E. Y. (1987). The prevalence of depressive symptoms in college students. Social Psychiatry, 22, 20-28.

Zeskind, P. S., \& Stephens, L. E. (2004). Maternal selective serotonin reuptake inhibitor use during pregnancy and newborn neurobehavior. Pediatrics, 113 , $368-375$. 